\title{
FIB-SEM NANOFABRICATION- A REVIEW
}

\author{
P. Ramesh ${ }^{1}$ \\ ${ }^{1}$ Department of Physics, SVR Degree College Macherla, Andhra Pradesh, India - 522426
}

\author{
K V L N Murthy ${ }^{2}$ \\ ${ }^{2}$ Department of Chemistry, SVR Degree College Macherla, Andhra Pradesh, India - 522426
}

Article DOI: $\underline{\text { https://doi.org/10.36713/epra5209 }}$

\begin{abstract}
Based on unique FIB and precision stage technology as well as a novel nanofabrication platform, the Raith FIBSEM is designed around the FIB as the primary patterning technique, supported by uncompromised SEM and laser interferometer stage capabilities. Such a FIB-centric state-of-the-art nanofabrication system allows scientists to take advantage of the unique capabilities afforded by focused ions. This paper discusses various capabilities and key strengths, based on exemplary applications from different nanopatterning areas.
\end{abstract}

\section{INTRODUCTION}

Over the last decades, focused ion beam (FIB) technology has evolved from a niche method into an extremely versatile technique for various application areas. FIB's great success has been accelerated in particular by the combination with scanning electron microscopy (SEM) in FIB-SEM microscopes. Today these FIB tools are found in most microscopy and analysis labs worldwide, where the FIB column is included as an add-on component for supporting sample preparation and basic patterning tasks. However, FIB is also an increasingly valuable tool for nanofabrication and rapid prototyping applications, providing direct resistless three-dimensional patterning and thus complementing other top-down lithography instrumentation. Even though patterning with ions has advanced very quickly to a high performance level, the SEM prioritized microscope architecture has limited it so far to more sophisticated applications.

\section{STRENGTHS AND LIMITATIONS OF EBL}

Today's state-of-the-art nanofabrication systems must deliver flexibility, stability over space and time, accuracy, and ease of use. These demands are fulfilled by EBL patterning: precise laser interferometer stage (LIS), best positional stability, enhanced beam current stability, excellent beam size deflection and accuracy are mandatory for these instruments. Moreover, software with industry standard GDSII designs, advanced beam control, navigation, and true automation are essential components of any dedicated nanofabrication system. These attributes have made EBL the most prominent fabrication method in the field of nanofabrication, achieving wafer scale patterning to nanometer accuracy in terms of placement and feature size in a reasonable time. However, EBL unfolds its strengths only in combination with other techniques used in the process chain. For transforming a substrate into a patterned substrate, EBL patterning is just one process step among many others, such as etching and lift-off. Furthermore, EBL is a superior method for 


\section{EPRA International Journal of Research and Development (IJRD) \\ Volume: 5 | Issue: 9 | September 2020 \\ - Peer Reviewed Journal}

lithography on flat substrates, where patterning of topographic samples can be complicated to achieve.

\section{EBL approach}

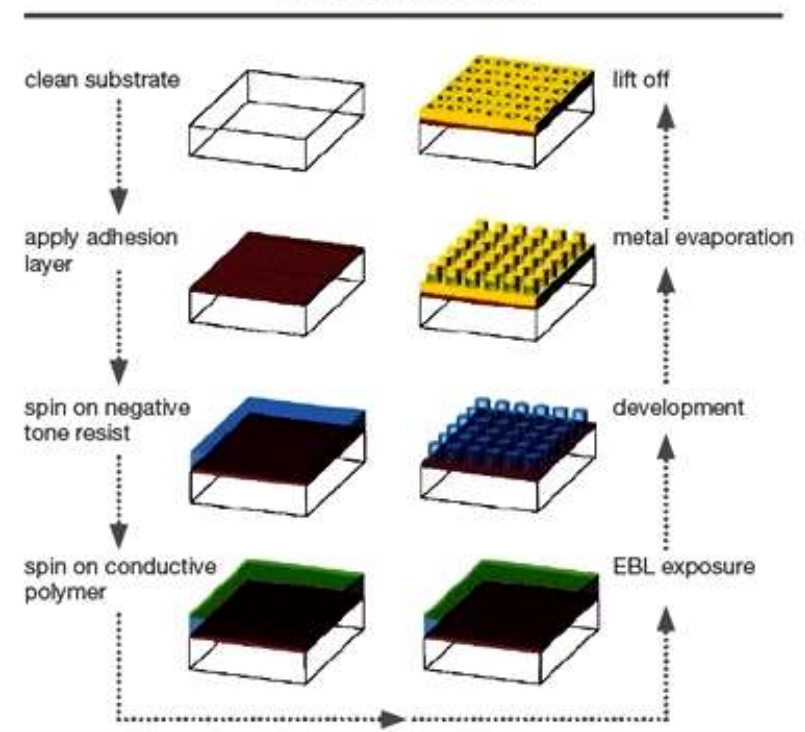

FIB approach

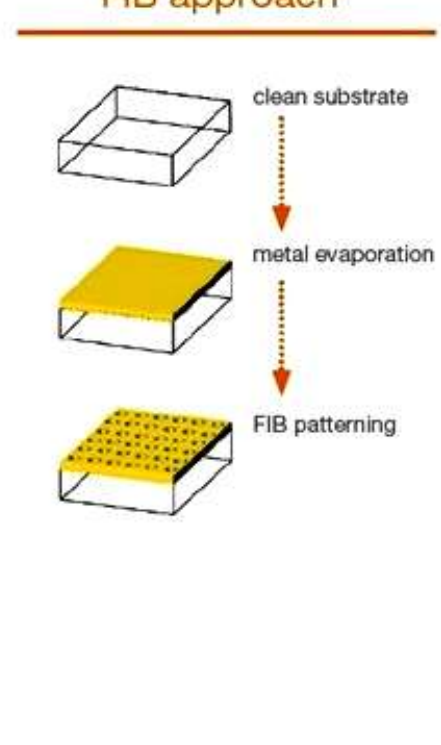

Figure 1: Number of steps for e.g. lift-off process is strongly reduced in case of FIB (right) compared to EBL approach (left).

Lean Nanofabrication: Core process steps addressed by FIB. The limitations of long process chain and atterning of topographic surfaces can easily be overcome by using a focused ion beam to structure substrates. FIB-SEM instruments are commonly used for 3 dimensional patterning - even over topographic samples. As another benefit of these systems, the resistless fabrication method allows direct patterning with far fewer process steps. As a consequence, development of new patterning processes can be achieved very fast thanks to in-situ process control.

Conventional FIB-SEM systems, designed for various microscopy and analysis applications in a SEM-centric setup, exhibit some limitations for nanofabrication applications. The FIB column is inclined with respect to the horizontal stage motion and the column and stage technology themselves, and this characteristic, in combination with the overall system and software platform, does not support advanced FIB nanopatterning. Long term highest-precision automated unattended or large-area processes demand a different class of technology and system architecture. These FIB capabilities plus the in-situ SEM process control unlock new pathways and help achieve results more rapidly for an increasing number of applications.

\section{FIB HARD MASKING OF LARGE AREA PATTERNS}

The first example shows a novel way of employing specific ion beam effects for the purpose of fast patterning. When patterning a substrate using a low ion dose, the $\mathrm{Ga}$ ions are implanted into the surface without even milling the top surface layer. Instead of creating topographic structures, the sample surface can be functionalized in a very controlled way by this $\mathrm{Ga}$ implantation with high lateral resolution. In a subsequent etching step, this functionalized top layer serves directly as a hard mask, resulting in a very simple and fast process. An example of the process workflow is illustrated in Figure 2. 


\section{EPRA International Journal of Research and Development (IJRD) \\ Volume: 5 | Issue: 9 | September 2020

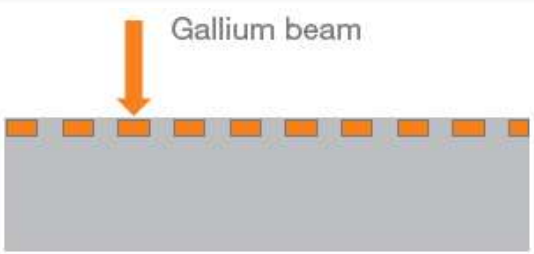

Specific $\mathrm{Ga}$ implants

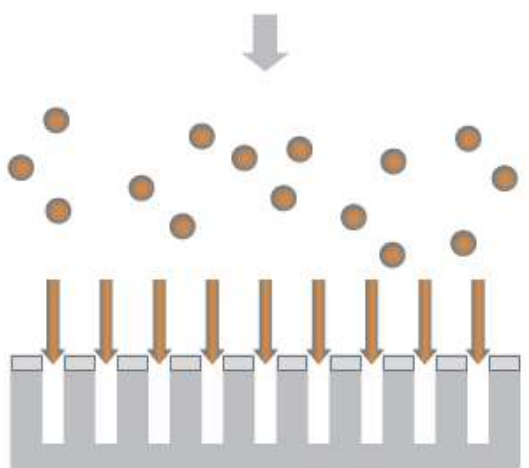

Hard mask for etching

Figure 2: Sketch of the functionalization process - after controlled Ga implantation trenches are etched into the substrate.

After functionalization of a diamond surface with Ga ions and subsequent fluorine plasma etching, a grating with about $400 \mathrm{~nm}$ line width and $50 \%$ duty cycle was fabricated (see Figure 3).

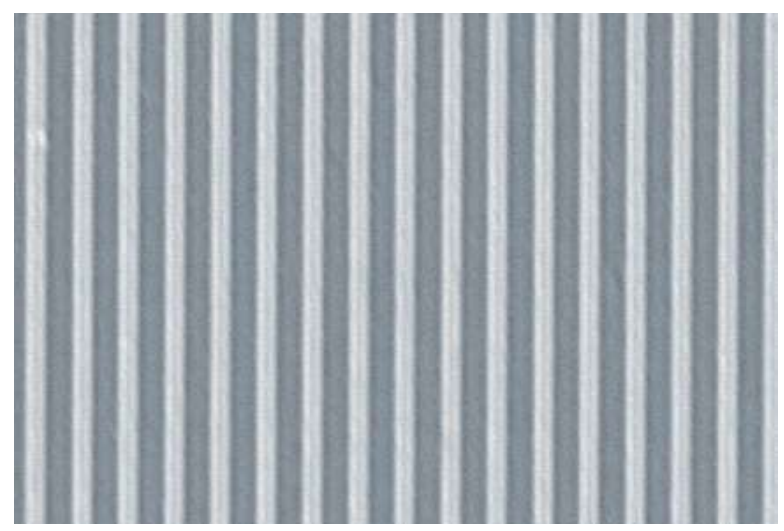

Figure 3: SEM viewgraph of the etched grating [1]

The complete design consists of multiple gratings with varying orientation. The overall structure extends over an area of $3 \times 4 \mathrm{~mm} 2$. The resulting pattern forms a diamond- like structure illustrated in the photograph of Figure 4. The different grating orientations generate different colors in the optical viewgraph. The functionalization step took $4 \mathrm{~h}$, which is comparatively fast. 


\section{EPRA International Journal of Research and Development (IJRD)}

Volume: 5 | Issue: 9 | September 2020

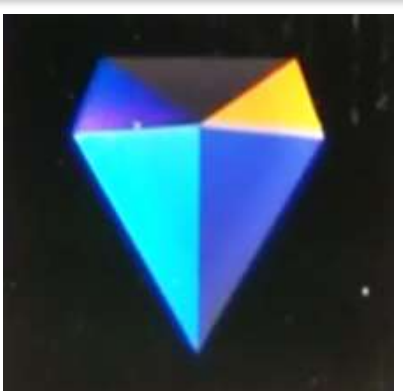

Figure 4: Photograph of the complete pattern after etching. Colors represent the different grating orientations[1]

\section{HIGH RESOLUTION FEATURES IN A LARGE ARRAY}

A FIB spot is usually not described by a pure Gaussian distribution, but instead exhibits significant beam tails outside the central spot. The contribution of these low dose beam tails becomes important for gas assisted processes or creates collateral damage close to, or even inside, the desired pattern. Although this dose is very small (10-2 - 10-5 of peak intensity), it can still have a significant influence on the result: the superposition of the beam tails at the edge of a pattern or between adjacent and dense patterns can lead to unwanted edge rounding or sample damage. Challenges for achieving small feature sizes and at the same time a large array for final measurements are given e.g. in the field of plasmonics, where large numbers of identical structures are designed with high pattern density.
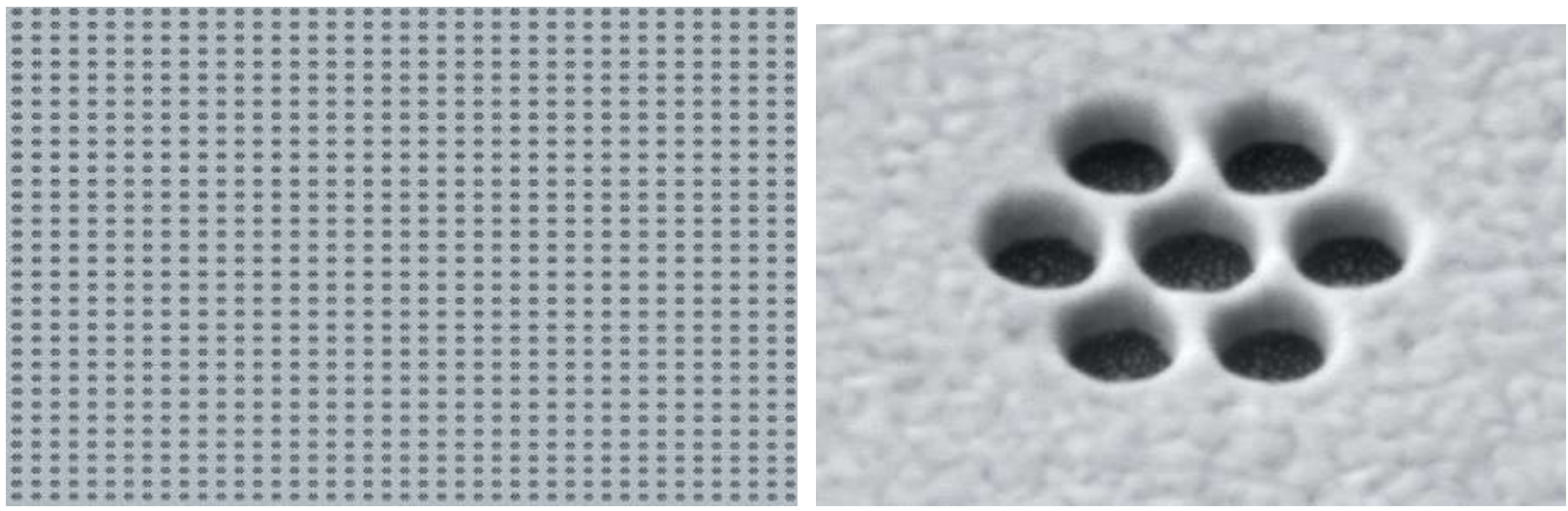

Figure 5: Oligomer structures within a $100-\mu \mathrm{m}$-wide array for plasmonics, showing excellent fidelity and reproducibility [2]

Figure 5 shows an array of 12,000 oligomers each consisting of 7 circles. Between two adjacent circles, the remaining strip of 80 -nm-thick gold has a width of only $30 \mathrm{~nm}$. The ability to create such a strip in gold is also good proof of excellent beam spot characteristics, specifically the record low beam tails, since the beam profile overlaps from several directions at this point. Finally, it is noted that the film consists of polycrystalline gold, which is even more difficult to pattern than single crystal gold films in terms of high resolution and reproducibility.

\section{DEVELOPMENT OF AN ADVANCED FIB PROCESS}

Fresnel zone plates (FZP) for X-rays require high accuracy, high aspect ratio and large area structures. In most cases they are fabricated withelectron beam lithography (EBL) based techniques plus plasma or wet etching, including extensive efforts in development of the complete nanofabrication process. For this reason, and because of the fact that many devices are made on membranes, a direct patterning technique like FIB offers significant benefits. Here we report on a FZP fabricated in a gold film (Figure 6). It is very difficult to achieve small feature size or high aspect ratio structures on such material, due to gold's high sputter yield and extreme sensitivity to collateral 


\section{EPRA International Journal of Research and Development (IJRD) \\ Volume: 5 | Issue: 9 | September 2020

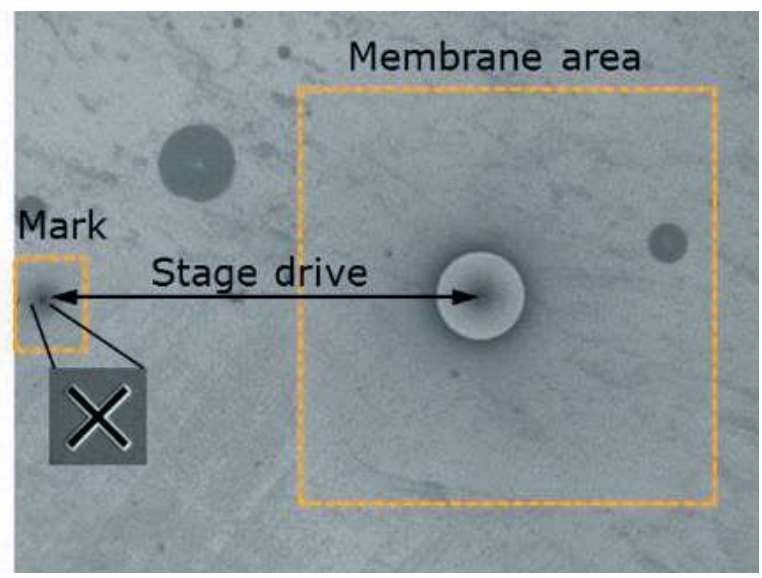

Figure 6: verview SEM image of the silicon nitride membrane with active area including gold zone plate and reference mark on bulk sample for automatic positioning correction [3]

beam damage. The pattern was fabricated by applying automated position correction with a reference mark on the bulk material far outside the field of view and the sensitive membrane area (Figure 6). This procedure significantly minimized the risk of membrane rupture.
The achieved total accuracy of better than $20 \mathrm{~nm}$ over $15 \mathrm{~h}$ thus includes several highly precise and "blind" drives of the laser interferometer stage to the alignment mark and back to the membrane.

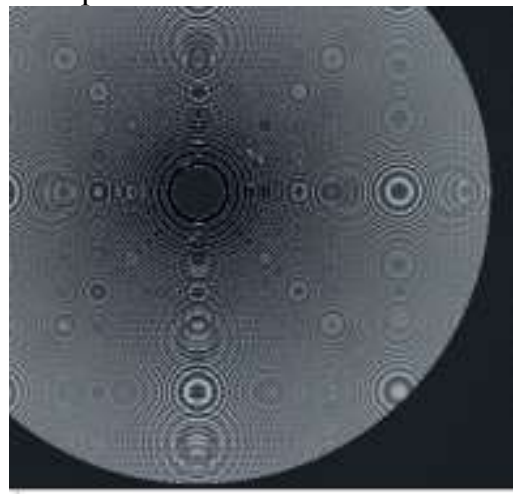

Figure 7: SEM viewgraph of the FZP [3]

Given the dimensions of the FZP, two parameters are the most crucial to investigate: the aspect ratio of the smallest gold zones, and the circularity of the complete zone plate. The first one was checked by crosssectioning and inspection at four different locations (top, bottom, left, right). The literature shows that an aspect ratio of even 5:1 is an excellent result for patterning of gold. Here the height of the outermost 100 $\mathrm{nm}$ zone was found to be almost unchanged (Figure 8). Verifying the circularity of $100 \mu \mathrm{m}$ to accuracy of much better than $100 \mathrm{~nm}$ is only possible by using a laser interferometer stage in combination with a navigation and metrology software. 


\section{EPRA International Journal of Research and Development (IJRD)}

Volume: 5 | Issue: 9 | September 2020

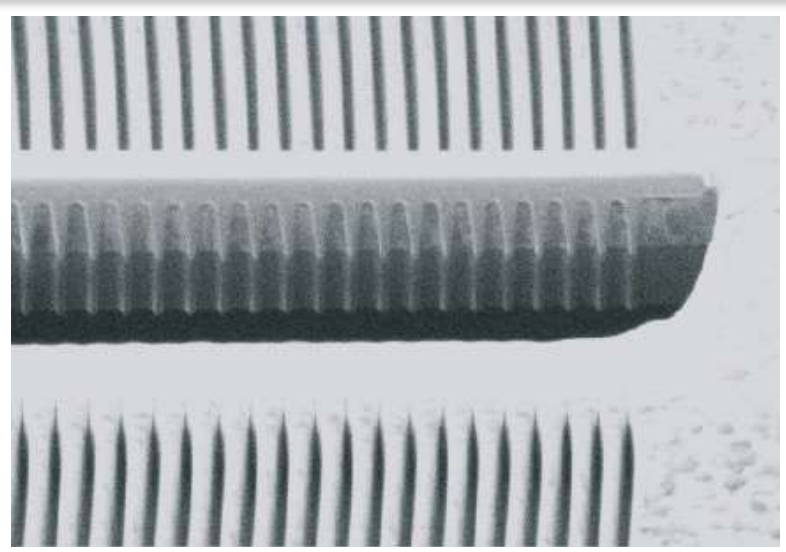

Figure 8: Ion beam cross-section image of outer zones after deposition of a protective and contrast enhancing layer of tungsten [3]

Making use of SEM based metrology, we set up high resolution e-beam line scans at 8 locations including diagonal positions (see Figure 9). We confirmed that the circularity differs by less than $20 \mathrm{~nm}$ from ideal, sufficient for excellent FZP performance. This has been confirmed by X-ray tests showing even better resolution than predicted from theory.

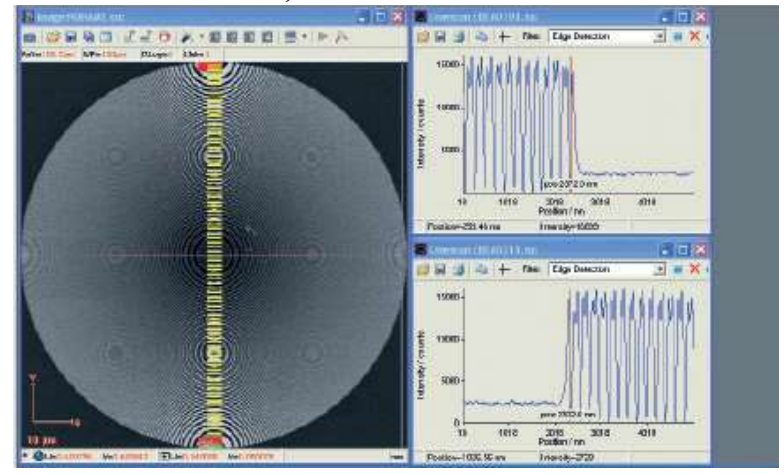

Figure 9: Example of e-beam line scan measurements to determine the diameter in a vertical direction [3]

\section{COMBINED EBL - FIB PROCESS AND QUALITY ASSURANCE AT WAFER SCALE}

Membrane based solid state nanopore devices for DNA sequencing and biomolecule analysis are of high interest from both scientific and commercial viewpoints. On the one hand, a feature size below 20 $\mathrm{nm}$ for the pore itself is mandatory; on the other, a large number of devices are used for research and, in particular, for commercial screening applications, which requires small batch true water scale production. 


\section{EPRA International Journal of Research and Development (IJRD)}

Volume: 5 | Issue: 9 | September 2020

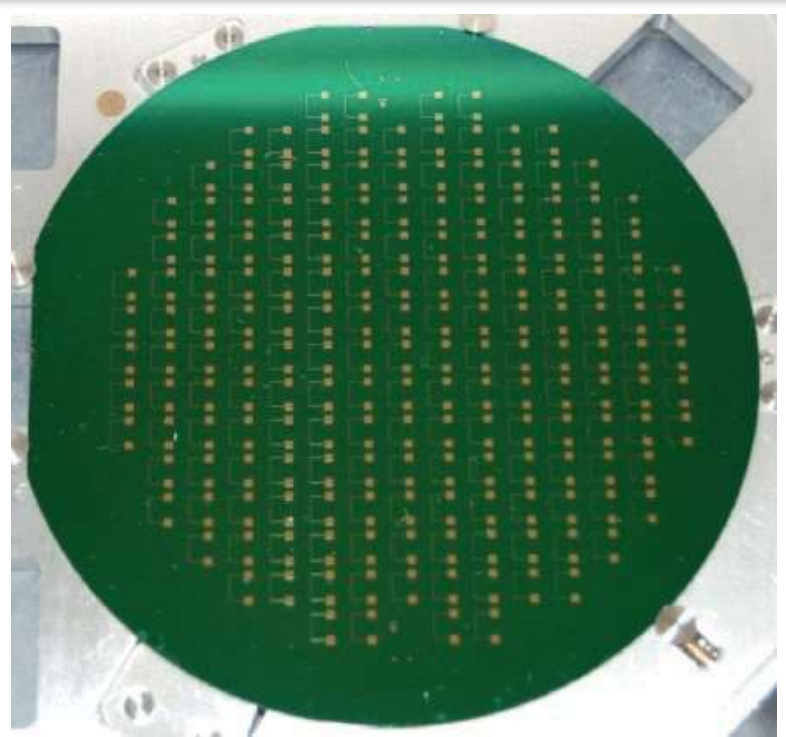

Figure 10: 4 inch wafer with 175 devices of connection pads and read-out electrodes [4]

Here we used a combination of EBL for creating dedicated read-out electrodes for 175 devices on a 4 inch wafer (Figure 10), and FIB for drilling the nanopore well aligned within the corresponding gap (Figure 11). The combination of both techniques, particularly for wafer scale step and repeat and highest resolution processing, is the only solution, since keeping each and every device perfectly in focus can be ensured by the automated height sensing function

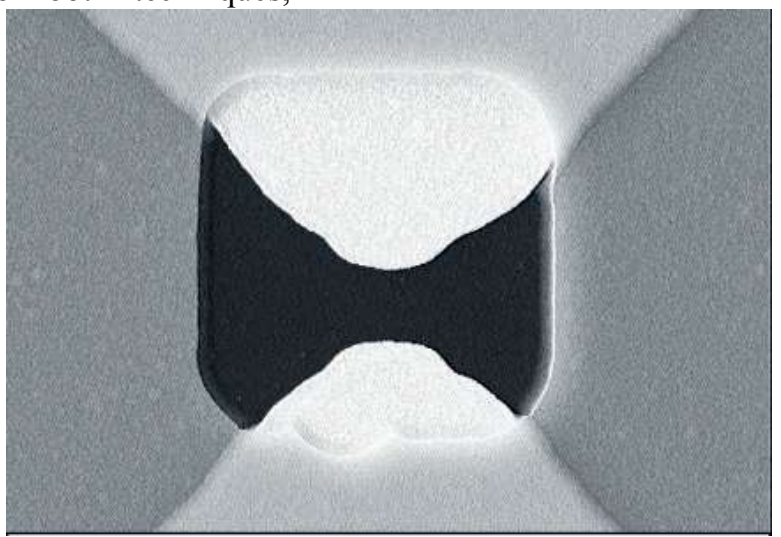

Figure 11: Read-out electrodes within window for placing the nanopore

in Raith systems. Therefore, we were able to achieve $20 \mathrm{~nm}$ pores in a $100 \mathrm{~nm}$ thick Si3N4 membrane across the wafer in a fully unattended way (Figure 12). This has been documented by electron beam quality assurance with a Raith EBL system which again is possible at wafer scale by means of automation 


\section{EPRA International Journal of Research and Development (IJRD)}

Volume: 5 | Issue: 9 | September 2020

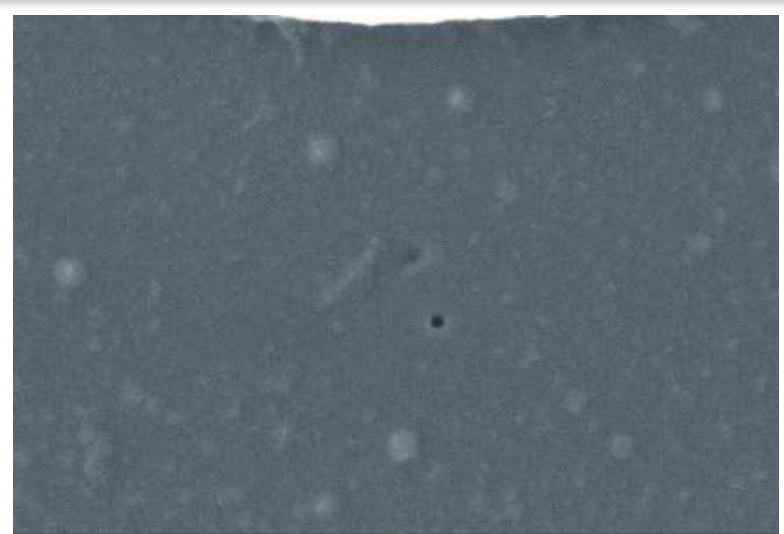

Figure 12: E-beam quality assurance of $20 \mathrm{~nm}$ nanopore in $100 \mathrm{~nm}$ thick Si3 $\mathrm{N4}$ membrane [4]

\section{TRUE 3D NANOSTRUCTURES AT ONCE OVER MM LENGTH}

Finally, structures for micro- and nanofluidic devices are good examples of cases where lateral and depth

definition is required to high precision levels and over extended areas. The process for creating a 3D microfluidic mixer channel of constant cross-sectional area while alternating between narrow and deep as well as shallow and wide (Figure 13) can be mastered in a unique approach using FIB nanofabrication. The design elements define the lateral shape and simultaneously the dose for the direct milling process controls the intentionally changing channel depth.

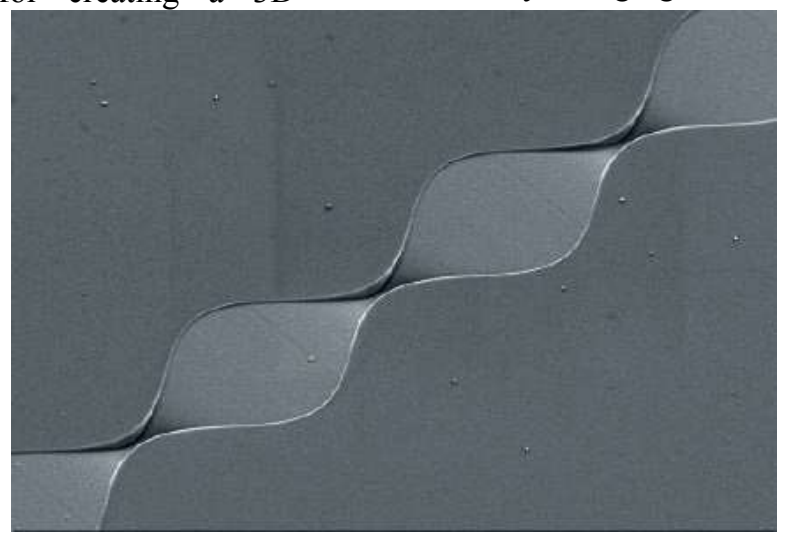

Figure 13: Part of a $1 \mathrm{~mm}$ long micro-fluidic mixer channel created by direct milling and stitching [5]

Moreover, employing the laser interferometer stage for stitching multiple base units enables a 1-mm-long channel to be achieved for real devices. This FIB milling process requires some extra care, given the enhanced sputter rate at the edge of subsequent write fields. Possible solutions include minimizing this characteristic by overlapping parts of the write fields with adapted dose distribution (Figure 14). Thus, a very smooth and 3D well defined channel is easily obtained directly in the substrate material. 


\section{EPRA International Journal of Research and Development (IJRD)}

Volume: 5 | Issue: 9 | September 2020

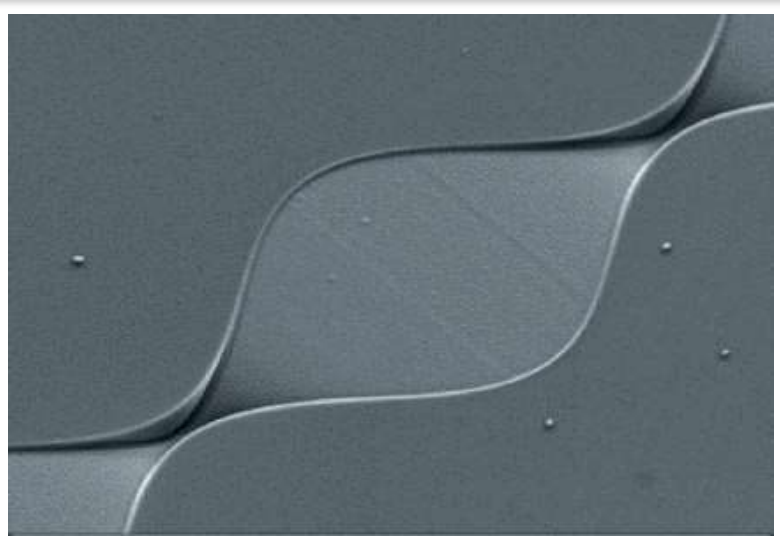

Figure 14: Advanced dose control and overlapping base units during stitching help overcome milling artifacts of adjacent write fields [5]

\section{REFERENCES}

Raith is happy to acknowledge its collaboration with the following partners:
1. Trinity College, Dublin
2. University of Stuttgart
3. Max-Planck-Institute, Stuttgart
4. Imperial College London
5. Argonne National Laboratory, Chicago 